DOI: 10.1002/aenm.((please add manuscript number))

Submitted to MATERIALS

Article type: Full Paper

\title{
Molecular weight dependence of exciton diffusion in poly(3-hexylthiophene)
}

Zarifi Masri, Arvydas Ruseckas, Evguenia V. Emelianova, Linjun Wang, Ashu K. Bansal, Andrew Matheson, Henrik T. Lemke, Martin M. Nielsen, Ha Nguyen, Olivier Coulembier, Philippe Dubois, David Beljonne, Ifor D. W. Samuel*

Z. Masri, Dr. A. Ruseckas, Dr. A. K. Bansal, Andrew Matheson, Prof. I. D. W. Samuel Organic Semiconductor Centre, SUPA, School of Physics and Astronomy, University of St. Andrews, North Haugh, St. Andrews KY16 9SS, UK

E-mail:idws@st-andrews.ac.uk

Dr. H. T. Lemke

Niels Bohr Institute, University of Copenhagen, 2100 Copenhagen, Denmark

Prof. M. M. Nielsen

Centre for Molecular Movies, Department of Physics, Technical University of Denmark, DK2800, Kgs. Lyngby, Denmark

H. Nguyen, Dr. O. Coulembier, Prof. P. Dubois, Dr. E. V. Emelianova, Dr. L.J. Wang, Prof.

D. Beljonne

Laboratory for Chemistry of Novel Materials, University of Mons, Place du Parc 20, B-7000

Mons, Belgium

Keywords: exciton diffusion; exciton-exciton annihilation; poly(3-hexylthiophene); molecular weight

We present a joint experimental and theoretical study of singlet exciton diffusion in spincoated P3HT films and its dependence on molecular weight. The results show that exciton diffusion is fast along the co-facial $\pi$ - $\pi$ aggregates of polymer chromophores and about 100 times slower in the lateral direction between aggregates. Exciton hopping between aggregates is found to show a subtle dependence on interchain coupling, aggregate size and Boltzmann statistics. Also a clear correlation is observed between the effective exciton diffusion coefficient, the degree of aggregation of chromophores and exciton delocalisation along the polymer chain, which suggests that exciton diffusion length can be enhanced by tailored synthesis and processing conditions. 


\section{Introduction}

Solution-processed conjugated polymers are promising materials for organic optoelectronic devices such as light-emitting diodes, field-effect transistors and solar cells. In solar cells, photons absorbed by the conjugated polymer generate tightly bound excitons which have to diffuse to an interface with an electron acceptor where they split into electron-hole pairs. Extraction of generated charges to the electrodes gives photocurrent. Two key parameters that determine solar cell performance are exciton diffusion length and charge carrier mobility. ${ }^{[1]}$ Exciton diffusion length determines the fraction of excitons that split into electron-hole pairs whereas charge carrier mobility determines the fraction of charges that reach an electrode for photocurrent generation. Exciton diffusion is particularly important in planar heterojunction solar cells where it limits the exciton dissociation rate but it also defines the slow phase of charge generation in bulk heterojunction solar cells which extends to $100 \mathrm{ps}^{[2,3]}$ and sets limits on the acceptable length scale of phase separation. Solar cells based on a bulk heterojunction of a semi-crystalline polymer poly(3-hexylthiophene) (P3HT) and a fullerene derivative PCBM show high internal quantum efficiencies of about 0.8 and have been studied extensively as model materials. ${ }^{[4]}$ Charge carrier mobility in P3HT shows strong dependence on film morphology which can be controlled by varying the molecular weight, solvent and processing conditions. ${ }^{[5-11]}$ In contrast, exciton diffusion has not been systematically studied and the reported values of the exciton diffusion length vary between 4 and $27 \mathrm{~nm} .{ }^{[12-16]}$

In this paper we investigate exciton diffusion in P3HT using two experimental techniques: surface quenching of the polymer fluorescence and exciton-exciton annihilation. We find that the diffusion coefficient measured by surface quenching for different molecular weight polymers vary by up to a factor of ten which is explained by different orientations of the co-facial $\pi-\pi$ stacks of polymer chromophores relative to the quenching surface. This is supported by X-ray diffraction results. We find that exciton diffusion occurs predominantly along the $\pi-\pi$ stacks with a much slower hopping between stacks and shows a clear 


\section{Submitted to ENERGY}

correlation with the fraction of aggregated chromophores and exciton delocalisation along the polymer chain. A theoretical model describing energy transfer in P3HT lamellae is developed and it further illustrates the subtle interplay of interchain coupling, aggregate size and Boltzmann statistics in the exciton hopping between the $\pi-\pi$ stacks.

\section{Results and Discussion}

\subsection{Surface quenching of fluorescence}

P3HT samples of high regioregularity (>98\%) and a range of number average molecular weights $M_{n}$ were used in this study and compared with poly(3-octylthiophene) (P3OT). We measured fluorescence quenching dynamics in spin-coated polymer films on planar $\mathrm{TiO}_{2}$ surface as described previously. ${ }^{[12]} \mathrm{We}$ assume that fluorescence intensity at time $t$ is proportional to the concentration of excitons $N(x, t)$ integrated over the thickness of the film, where $x$ is the distance from the interface. The results were modeled using a one-dimensional diffusion equation

$$
\frac{\partial N}{\partial t}=D \frac{\partial^{2} N}{\partial x^{2}}-k(t) N
$$

where $D$ is the exciton diffusion coefficient in the direction perpendicular to the substrate and $k(t)$ is the exciton decay rate in the film of the same thickness but on a non-quenching substrate (fused silica). The boundary conditions applied were that the population of excitons goes to zero at the film- $\mathrm{TiO}_{2}$ interface, whereas the film-vacuum and film-fused silica interfaces were assumed to perfectly reflect excitons. This gives a lower limit for the diffusion coefficient as a finite quenching rate at the film- $\mathrm{TiO}_{2}$ interface would result in a larger diffusion coefficient to achieve the same amount of quenching. Figure 1 shows the fluorescence decays in polymer films spin-coated from p-xylene solution on $\mathrm{TiO}_{2}$ and fused 


\section{ENERGY \\ Submitted to MATERIALS}

silica substrates with the $D$ values obtained by fitting the decays. We obtained $D=2 \times 10^{-4}$ $\mathrm{cm}^{2} \mathrm{~s}^{-1}$ in P3HT films of medium molecular weight $M_{n}=13800 \mathrm{gmol}^{-1}$ and $D=1 \times 10^{-4} \mathrm{~cm}^{2} \mathrm{~s}^{-1}$ in P3OT films with $M_{n}=13500 \mathrm{gmol}^{-1}$. We also performed a similar surface quenching experiment using tethered $\mathrm{C}_{60}$ as a quencher ${ }^{[17]}$ and obtained $D$ values which were two times bigger. These results are given in Supporting Information (SI) in Figure S1. There is a nonnegligible spectral overlap between the fluorescence of P3HT and absorption of tethered $\mathrm{C}_{60}$ which results in a longer quenching distance by the Förster resonance energy transfer and this slightly overestimates values of $D$ measured using $\mathrm{C}_{60}$ as a quencher. The $D$ values obtained from quenching on $\mathrm{TiO}_{2}$ substrates and from quenching on tethered $\mathrm{C}_{60}$ give the lower and upper limits for the diffusion coefficient respectively.

We found that P3HT films of high $M_{n}=76000 \mathrm{gmol}^{-1}$ showed substantially higher diffusion coefficient of $2 \times 10^{-3} \mathrm{~cm}^{2} \mathrm{~s}^{-1}$ (data not shown) but consistent with previous measurements on high $M_{n}$ P3HT. ${ }^{[12]}$ This difference between films of different $M_{n}$ could be explained by different orientation of the self-organised lamellae relative to the substrate. ${ }^{[10,18,19]}$ In order to test this, grazing-incidence X-ray diffraction (XRD) experiments were performed on $80 \mathrm{~nm}$ thick films spin-coated on fused silica substrates in reflection geometry. Spin-coated films of medium $M_{n}$ P3HT and P3OT show intense and narrow out-of-plane (100) XRD peaks which indicate that lamellae are preferentially oriented perpendicular to the substrate (edge-on orientation, see Figure 2). From these data we determine the inter-stack distance $r_{p}=1.65 \mathrm{~nm}$ for P3HT and $r_{p}=2.03 \mathrm{~nm}$ for P3OT. In addition, the (010) diffraction peaks of the $\pi$ - $\pi$ stacks are observed in P3HT in the plane of the film and out-of-plane which give the inter-chain distance of $0.38 \mathrm{~nm}$ in $\pi-\pi$ stacks (shown schematically in Fig. 1). These results are consistent with previous reports on medium $M_{n}$, highly regio-regular P3HT. ${ }^{[10,18]}$ In case of edge-on lamellae fluorescence quenching by $\mathrm{TiO}_{2}$ measures exciton diffusion coefficient $D_{p}$ in the direction perpendicular to the $\pi$ - $\pi$ stack and lower $D_{p}$ values found in P3OT are consistent 


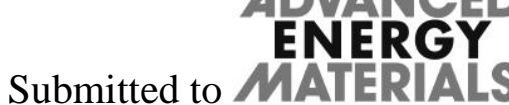

with larger inter-stack distance. In contrast, recent reports showed that films spun from high $M_{n}$ P3HT show both, edge-on and face-on orientations of $\pi-\pi$ stacks in films thicker than a few nm. ${ }^{[18,19]}$ In that case fluorescence quenching by $\mathrm{TiO}_{2}$ returns a $D$ value which is between $D_{p}$ and a faster diffusion along the stack $D_{z}$ driven by strong dipole-dipole interactions between $\pi$-stacked P3HT chromophores. The fraction of different orientations is unknown, therefore such measurements give only a lower limit for $D_{z}$.

\subsection{Exciton-exciton annihilation}

In order to investigate exciton diffusion and how it is affected by molecular weight, we studied singlet-singlet exciton annihilation. This process occurs at high excitation densities when two excitons can meet on the same chromophore and annihilate to form a higher energy exciton which can then either relax to the lowest energy excited state or dissociate into an electron-hole pair. As a result, one or both excitons are lost per annihilation event. The exciton concentration $N$ is described by a rate equation

$$
\frac{d N}{d t}=-k N-\gamma N^{2}
$$

where $k$ is the exciton decay rate in the absence of annihilation and $\gamma$ is the annihilation rate. We then deduce the time-dependence of $\gamma$ by measuring at two excitation densities:

$\gamma=\frac{1}{N_{2}}\left\{\frac{d N_{1} / d t}{N_{1}}-\frac{d N_{2} / d t}{N_{2}}\right\}$

where $N_{l}(t)$ is the exciton density following excitation at low intensity and $N_{2}(t)$ is the exciton density following higher intensity excitation. This approach is similar to the one described by 


\section{Submitted to ENERGY}

Gulbinas et al. ${ }^{[20]}$ except that we use the fluorescence decay at a very low excitation density as a reference. Figure 3 shows exciton population decays measured using different excitation pulse energies which result in different initial exciton concentration and $\gamma(t)$ calculated using Eq (3). In order to increase the time resolution and to reduce the noise associated with differentiation of the experimental data, we first fitted the experimental decays with a threeexponential decay function convolved with the instrument response and used the fits to calculate $\gamma(t)$. We observe a decrease of the $\gamma$ value in the first 20 ps after the excitation pulse and use an equation derived for diffusion-limited annihilation in the case of preferentially 1D diffusion with a spherical annihilation volume with a radius $r_{a}$ to fit $\mathrm{it}^{[21]}$

$$
\gamma=8\left(D_{z} D_{p}\right)^{1 / 2} r_{a}+\pi r_{a}^{2}\left(\frac{2 D_{z}}{\pi t}\right)^{1 / 2}
$$

The data shown in Figure 3(d) obtained from transient absorption measurements with $100 \mathrm{fs}$ time resolution fit much better with the anisotropic exciton diffusion model than with an isotropic model. The fit gives $D_{p}=2 \times 10^{-4} \mathrm{~cm}^{2} \mathrm{~s}^{-1}$ and $D_{z}=2 \times 10^{-2} \mathrm{~cm}^{2} \mathrm{~s}^{-1}$ for P3HT film with $M_{n}=76000 \mathrm{gmol}^{-1}$. The time resolution of fluorescence measurements ( 2 ps), however, was not sufficient to obtain $D_{p}$ and $D_{z}$ values independently for polymers of other $M_{n}$. Instead, we determine an effective diffusion coefficient $D_{\text {eff }}=\left(D_{z} D_{p}\right)^{1 / 2}$ from the time-independent term in Equation 4.

Previous studies showed that charge carriers are generated in polythiophene films with dense packing in addition to excitons and they can annihilate excitons. ${ }^{[22-24]}$ To study a possible contribution from exciton-charge annihilation we measured intensity-dependent fluorescence decays at different excitation photon energies of $2.2 \mathrm{eV}, 2.45 \mathrm{eV}$ and $3.1 \mathrm{eV}$. Charge carrier yield in neat P3HT films increases with photon energy, ${ }^{[25]}$ so the apparent $\gamma$ value would also 


\section{Submitted to ENERGY}

increase if exciton-charge annihilation is active. The $\gamma$ values however were independent of photon energy, so we rule out a contribution from exciton-charge annihilation at the excitation densities used in our experiments $\left(10^{18}-10^{19} \mathrm{~cm}^{-3}\right)$. It is worth noting that we indeed observe an increase of $\gamma$ values at the highest excitation densities at long times $t>300 \mathrm{ps}$ (see Figure 4) which is likely to include a contribution from exciton-charge annihilation as more charges can be generated from a higher energy exciton state populated by exciton-exciton annihilation. In order to avoid this charge generation effect and also to avoid the strong time dependence at early times, we take the average of $\gamma$ from 100 ps to $300 \mathrm{ps}$ giving $\gamma=7.1 \times 10^{-9} \mathrm{~cm}^{3} \mathrm{~s}^{-1}$ for data in Figure 4(c) and $\gamma=5.4 \times 10^{-9} \mathrm{~cm}^{3} \mathrm{~s}^{-1}$ for data in Figure 4(d). Using the previously reported value $r_{a}=1.8 \mathrm{~nm},{ }^{[12]}$ we obtain $D_{\text {eff }}=4.9 \times 10^{-3} \mathrm{~cm}^{2} \mathrm{~s}^{-1}$ and $D_{\text {eff }}=3.8 \times 10^{-3} \mathrm{~cm}^{2} \mathrm{~s}^{-1}$ for a P3HT film with $M_{n}=13800 \mathrm{gmol}^{-1}$ and $M_{n}=40000 \mathrm{gmol}^{-1}$ respectively.

\subsection{Correlation of exciton diffusion with aggregation and exciton bandwidth}

Figure 5(a) shows the effective diffusion coefficients $D_{\text {eff }}$ obtained from exciton-exciton annihilation measurements described in the previous section for different $M_{n}$ P3HT films made from chloroform solution and from chlorobenzene solution. An increase of $D_{\text {eff }}$ is observed from low to medium $M_{n}\left(4000-40000 \mathrm{gmol}^{-1}\right)$ whereas the polymer with high $M_{n}$ (76000 gmol $^{-1}$ ) shows slightly lower $D_{\text {eff }}$ than the medium $M_{n}$ polymers. The roomtemperature optical absorption spectra of the $\mathrm{P} 3 \mathrm{HT}$ films in this study were measured and are shown in Figure 6. The films made from chloroform solution show relatively featureless absorption spectra [Figure 6(a)] whereas the films made from chlorobenzene solution display more structured spectra with peaks resolved at $\sim 2.05 \mathrm{eV}$ and $\sim 2.23 \mathrm{eV}$ [Figure 6 (b)]. The prominent features seen in the absorption spectra of films made from chlorobenzene solution are due to the more crystalline film microstructure attained as the higher boiling point solvent 


\section{ENERGY \\ Submitted to MATERIALS}

affords more time for the polymer chains to self-organize and align themselves to form $\pi$ stacked aggregates.

Following previous work of Clark et al. ${ }^{[11]}$ we determine the fraction of the aggregated P3HT chromophores by subtracting the contribution of the amorphous phase in the absorption spectra which is assumed to show a solution-like absorption spectrum. The oscillator strength of planar chains in the aggregates was assumed to be $39 \%$ higher than that of non-planar chains in the amorphous phase. ${ }^{[11]}$ The aggregate fraction obtained for different molecular weights in two solvents is displayed in Figure 5(b) and it shows a clear correlation with the effective diffusion coefficient.

The analysis of the absorption spectra shown in Figure 6 also allows us to extract the free exciton bandwidth $W$ in the aggregate using a weakly-interacting $\mathrm{H}$-aggregate model developed by Spano and coworkers. ${ }^{[26]}$ The exciton bandwidth is estimated from the ratio of the $0-0$ and 0-1 absorbance peaks using the following expression ${ }^{[7,9,26]}$ :

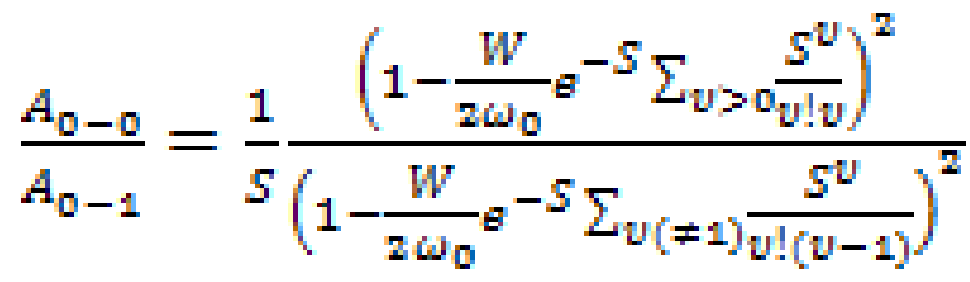

where $S$ is the Huang-Rhys factor and $\omega_{0}$ is the energy of the main vibrational mode coupled to the electronic transition. For polythiophenes, the $\mathrm{C}=\mathrm{C}$ symmetric stretch at around 1400 $\mathrm{cm}^{-1}$ is assumed to dominate the coupling. In order to compare with other previous work, ${ }^{[11]} \mathrm{a}$ Huang-Rhys factor of 1 has been used for the intrachain exciton. Using the experimental absorbance ratios, the free exciton bandwidths $W$ were calculated and are shown in Figure 5(c). The exciton bandwidth and the strength of interchain interactions are related by the 
extent of exciton delocalisation i.e. conjugation length. It has been shown by quantumchemical calculations that increasing the exciton delocalisation along the polymer chain reduces $W \cdot{ }^{[27,28]}$ Figure 5(c) shows that films spun from chloroform generally display higher $W$ and hence shorter conjugation lengths while films spun from chlorobenzene exhibit lower $W$ suggesting longer conjugation lengths. The dependence on $M_{n}$ suggests that the longest conjugation length is attained in the medium $M_{n}$ P3HT films.

From the results in Fig. 5 we can conclude that the increased chain aggregation and exciton delocalisation enhances exciton diffusion. This can partially be explained by the observation that excitons diffuse faster along the aggregated stack. The effect of exciton delocalisation is discussed in the next section.

\subsection{Theoretical modelling}

To explain the experimentally measured effect of molecular weight on exciton diffusion, we have developed a theoretical model for exciton diffusion in P3HT lamellae. The relatively large electronic couplings between the conjugated polymer chains within the stacks yield twodimensional (2D) excitons delocalised over multiple polymer chains (the actual exciton size depends on the interplay between intermolecular excitonic interactions and the amount of intramolecular disorder and varies across the density of states, see the inset to Figure 7(a)). These $2 \mathrm{D}$ excitons can then jump from stack to stack according to a Förster-like hopping mechanism. ${ }^{[29]}$ In our model, we first build the exciton Hamiltonian and obtain the intrastack exciton states by diagonalizing the Hamiltonian within each stack. Then we calculate the stack-to-stack hopping rates between the resulting $2 \mathrm{D}$ excitons and provide a qualitative analysis of the associated diffusivity using a simple variable range hopping approximation. The model was applied for two different lamella structures built from ideal stacks of P3HT 
molecules containing 8 and 40 repeated monomers (1360 and $6800 \mathrm{gmol}^{-1}$, respectively), as in Figure 1(c).

The exciton energy band was built by calculating the eigenvalues of the electronic Hamiltonian, $H$, where off-diagonal elements $H_{\mathrm{ij}}$ representing the electronic couplings between molecules $i$ and $j$ in a given stack are given by: $H_{i j}=\frac{1}{4 \pi \varepsilon_{0}} \sum_{l, m} Q_{l}{ }^{i} Q_{m}{ }^{j} F\left(r_{l m}\right), i, j=1$, $\ldots, N_{P}$. The Mataga-Nishimoto potential was used to depict the electron interaction potential $F$, the atomic transition charge densities $Q$ have been computed through correlated coupled cluster calculations with single and double excitations (CCSD) using the INDO Hamiltonian, and $\varepsilon_{0}$ is the vacuum permittivity. ${ }^{[30,31]}$ The on-site energies (diagonal elements) $H_{i i}$ were sampled from a Gaussian distribution with standard deviation $\sigma=0.1 \mathrm{eV}$ to account for intrastack static energetic (conformational) disorder. Assuming a simple Gaussian-like shape for the exciton absorption and emission $\operatorname{spectra}^{[32]}$ the Förster rate for exciton hopping between stacks is:

$$
\begin{aligned}
& k_{F}=\frac{2 \pi}{\hbar}<\sum_{k, k^{\prime}} P_{k} J_{k k^{\prime}}{ }^{2} F_{k k^{\prime}}>,[6] \\
& F_{k k^{\prime}}=\frac{1}{\sqrt{2 \pi} \sigma_{h}} \exp \left[-\frac{\left(E_{k}-E_{k^{\prime}}-\Delta_{s s}\right)^{2}}{2 \sigma_{h}{ }^{2}}\right], \text { [7] }
\end{aligned}
$$

where brackets denote averaging over disorder (typically a few thousand realizations), $J_{k k^{\prime}}$ is the electronic coupling between the $k$-th exciton state of the donor stack and $k^{\prime}$-th exciton state of the acceptor stack $\left(|k\rangle=\sum_{i} \varphi_{k i}|i\rangle\right.$ with $\varphi_{k i}$ the $i$-th component of the $k$-th eigenfunction obtained by diagonalizing the Hamiltonian $H$ over the isolated stacks and $\left.J_{k k^{\prime}}=\left\langle k|H| k^{\prime}\right\rangle\right), F_{k k^{\prime}}$ defines the spectral overlap between $k$-th state emission and $k^{\prime}$-th state 


\section{ENERGY \\ Submitted to MATERIALS}

absorption, $\Delta_{s s}$ is a fixed Stokes shift (set to $0.2 \mathrm{eV}^{[33]}$ ), $P_{k}$ the Boltzmann weighted population

of $k$-states in the donor stack, and $\sigma_{h}=\sqrt{2 k T \Delta_{s s}}$.

In Figure 7(a), we show the stack-to-stack energy transfer Förster-like rates for two different pairs of identical stacks (1360 and $6800 \mathrm{gmol}^{-1}$, or 8 and 40 repeated monomers, correspondingly) as a function of the stack size (i.e., the number of polymer chains in the stack). Independently on the stack size, the Förster hopping rate $k_{\mathrm{F}}$ is approximately 3.5 times larger for stacks with the higher molecular weight. This trend results from the conjunction of the fact that $\mathrm{P} 3 \mathrm{HT}$ chains organize into $\mathrm{H}$-aggregates featuring low-energy weakly dipole allowed electronic excitations and the presence of a rather large (compared to the excitonic bandwidth) conformational energetic disorder. The consequence of the latter is that only a few of the lowest energy states have significant Boltzmann weight at room temperature. The consequence of the former is that these states are optically dark in the absence of disorder and should therefore be inefficient for singlet hopping in the Förster limit (though this is somewhat relaxed in our calculations that go beyond the point dipole approximation). However, the presence of disorder translates into a symmetry breaking effect heading towards (Anderson) localization of the exciton wavefunctions (in the limit of large disorder, the excitations are localized on a single chain). Such localization effects are more pronounced for longer conjugated segments because they have smaller free electronic bandwidth. This can be better appreciated from the insert to Figure 7(a) where the participation ratios ( $\left.P R=\sum_{i}\left|\varphi_{k i}\right|^{4}\right)$ for the lowest 5 exciton states are portrayed (the PR tends to 1 for complete localization and to 0 for complete delocalisation). It is clear from Figure 7(a) that the PR increases from $M_{n}=1360 \mathrm{gmol}^{-1}$ to $M_{n}=6800 \mathrm{gmol}^{-1}$, where the lowest exciton state is almost confined on a single polymer chain (PR 1). As a result, the channel (state) input to the Förster energy transfer rate between stacks is larger for longer polymer chains (see Figure 7(b)). In other 


\section{Submitted to}

words, the detrimental effect of the formation of non-emissive H-aggregate-like low-lying excited states is largely suppressed in going to large molecular weights.

The diffusion coefficients for stack-to-stack energy transport $D_{p}$ can be inferred from the rates above by using a simple variable-range hopping model and is given by the ratio of the squared displacement of exciton with respect to time. The calculated values of $D_{p}=2.0 \times 10^{-4} \mathrm{~cm}^{2} \mathrm{~s}^{-1}$ for $M_{n}=1360 \mathrm{gmol}^{-1}$ and $D_{p}=6.2 \times 10^{-4} \mathrm{~cm}^{2} \mathrm{~s}^{-1}$ for $M_{n}=6800 \mathrm{gmol}^{-1}$ are in good agreement with the lower and upper values of $D_{p}=2 \times 10^{-4} \mathrm{~cm}^{2} \mathrm{~s}^{-1}$ and $D_{p}=4 \times 10^{-4} \mathrm{~cm}^{2} \mathrm{~s}^{-1}$ obtained by surface quenching. The result shown in Figure 7(a) suggests that faster inter-stack diffusion $D_{p}$ is achieved for smaller numbers of molecules in the stack. This means that films with smaller aggregate fraction may display higher $D_{p}$ which thus can explain why $D_{\text {eff }}$ shows just a weak dependence on $M_{n}$.

\section{Conclusions}

The design of organic optoelectronic devices could benefit greatly from a comprehensive understanding of the factors that affect exciton transport. In this paper, we have presented experimental results that show the influence of molecular weight on intermolecular interactions and exciton diffusion. We find that exciton diffusion is correlated with the aggregate fraction and extent of exciton delocalisation along the chain. The highest exciton diffusion coefficients were obtained in the medium molecular weight samples which have the largest fraction of aggregates and extensive exciton delocalisation. We have also developed a model to predict the effect of molecular weight on exciton diffusion by quantifying the Förster rate of stack-to-stack energy transfer in two model lamellae of molecular weight 1360 and $6800 \mathrm{gmol}^{-1}$. In this model, the higher molecular weight polymer $\left(6800 \mathrm{gmol}^{-1}\right)$ was found to have higher exciton diffusion coefficient. The model suggests that the increased exciton delocalisation along the polymer chain in larger molecular weight polymers has a 
beneficial effect on inter-stack diffusion. Interestingly, despite smaller electronic interactions between neighboring polymer chains in the stack at large $M_{n}$, larger molecular weights also maximize the intra-stack exciton diffusion process owing to a long-range transfer mechanism. ${ }^{[34]}$ The model further illustrates the subtle interplay of interchain coupling, aggregate size and Boltzmann statistics in the energy transfer process. These results suggest that by developing materials with increased crystallinity and conjugation length, exciton diffusion length can be increased.

\section{Experimental Section}

Materials: The P3HTs studied here were obtained as described in Ref.35. High $M_{n}$ P3HT $\left(76000 \mathrm{gmol}^{-1}\right)$ was obtained from Merck Chemicals. All materials had regioregularity of $98 \%$ or higher and comparable dispersity values $\left(\bigoplus_{M}\right)$ except for the high molecular weight material (see Table 1).

Film preparation: Solutions were prepared in chloroform and chlorobenzene and stirred overnight in a $\mathrm{N}_{2}$-filled glove box at $50^{\circ} \mathrm{C}$ and $90^{\circ} \mathrm{C}$ respectively. Films were spin-coated onto fused silica substrates. All substrates were first cleaned via ultrasonication for 15 minutes with acetone and propan-2-ol. The films were spin-coated in the glove box using spin speeds of around 1500-2000 rpm. No heat treatment was performed on these films. Films made for transient absorption measurements were sandwiched and sealed between two fused silica substrates in the glove box. For measurements of fluorescence quenching by $\mathrm{TiO}_{2}$, polymer films were spin-coated from p-xylene solution on $\mathrm{TiO}_{2}$ substrates prepared by vacuum deposition. ${ }^{[12]}$ Substrates with tethered C60 used for other fluorescence quenching experiments were prepared as described previously. ${ }^{[17]}$

Measurements: The absorbance spectra of the films were measured using a Varian Cary 300 UV-Vis Spectrophotometer. Film thicknesses were measured with a Veeco Dektak 150 surface profiler and also estimated from the absorbance. For films spin-coated on $\mathrm{TiO}_{2}$, film 
thicknesses ranged from 12-14 nm. Film thicknesses for fluorescence measurements varied between 23 to $74 \mathrm{~nm}$. Fluorescence decays at low excitation density were measured using 100 fs pulses at $3.1 \mathrm{eV}$ excitation with $80 \mathrm{MHz}$ repetition rate and a Hamamatsu C6860 synchroscan streak camera for detection. For exciton-exciton annihilation in fluorescence and transient absorption measurements the $100 \mathrm{fs}$ laser pulses at a repetition rate of $5 \mathrm{kHz}$ were used. All fluorescence measurements were performed under a vacuum of $10^{-4}$ mbar. The energy of the laser pulses was controlled with neutral density filters. The excitation spot was measured with a LaserCam 3D beam profiler and found to be an ellipse with the major and minor diameters of $290 \mu \mathrm{m}$ and $215 \mu \mathrm{m}$ in fluorescence measurements. In transient absorption measurements, the ellipse size of the excitation spot was $2.22 \mathrm{~mm}$ by $1.64 \mathrm{~mm}$. A film thickness of $170 \mathrm{~nm}$ and a probe of $1.0 \mathrm{eV}$ were used for transient absorption measurements.

\section{Supporting Information}

Supporting Information is available online from the Wiley Online Library or from the author.

\section{Acknowledgements}

Z.M. is grateful to the Government of Brunei Darussalam for financial support. Work at St Andrews is supported by the Engineering and Physical Sciences Research Council of the UK and by the European Research Council of the European Union. The P3HTs preparation has been supported by the European Commission and Région Wallonne FEDER program (Materia-Nova) and OPTIMAT program of excellence, by the Interuniversity Attraction Pole programs of the Belgian Federal Science Policy Office (PAI 6/27 and 7/05) and by FNRSFRFC. O.C. is Research Associate for the F.R.S.-FNRS in Belgium. The authors gratefully acknowledge Alexander J. Ward for preparation of tethered $\mathrm{C}_{60}$ and Dr. S. Clément and Dr. G. Grancharov for fruitful methodological discussions. 


\section{REFERENCES}

[1] R. R. Lunt, J. B. Benziger, S. R. Forrest, Adv Mater, 2010, 22, 1233.

[2] J. Kirkpatrick, P. E. Keivanidis, A. Bruno, F. Ma, S. A. Haque, A. Yarstev, V. Sundstrom, J. Nelson, J Phys Chem B, 2011, 115, 15174.

[3] A. Ruseckas, P. E. Shaw, I. D. W. Samuel, Dalton T, 2009, 10040.

[4] M. T. Dang, L. Hirsch, G. Wantz, Adv Mater, 2011, 23, 3597.

[5] A. Zen, J. Pflaum, S. Hirschmann, W. Zhuang, F. Jaiser, U. Asawapirom, J. P. Rabe, U. Scherf, D. Neher, Adv Funct Mater, 2004, 14, 757.

[6] J. F. Chang, J. Clark, N. Zhao, H. Sirringhaus, D. W. Breiby, J. W. Andreasen, M. M. Nielsen, M. Giles, M. Heeney, I. McCulloch, Phys Rev B, 2006, 74, 115318.

[7] J. Clark, C. Silva, R. H. Friend, F. C. Spano, Phys Rev Lett, 2007, 98.

[8] O. G. Reid, J. A. N. Malik, G. Latini, S. Dayal, N. Kopidakis, C. Silva, N. Stingelin, G. Rumbles, J. Polym. Sci. Pt. B-Polym. Phys., 2011, 50, 27.

[9] C. Scharsich, R. H. Lohwasser, M. Sommer, U. Asawapirom, U. Scherf, M. Thelakkat, D. Neher, A. Kohler, J. Polym. Sci. Pt. B-Polym. Phys., 2012, 50, 442.

[10] H. Sirringhaus, P. J. Brown, R. H. Friend, M. M. Nielsen, K. Bechgaard, B. M. W. Langeveld-Voss, A. J. H. Spiering, R. A. J. Janssen, E. W. Meijer, P. Herwig, D. M. de Leeuw, Nature, 1999, 401, 685.

[11] J. Clark, J.-F. Chang, F. C. Spano, R. H. Friend, C. Silva, Appl Phys Lett, 2009, 94, 163306.

[12] P. E. Shaw, A. Ruseckas, I. D. W. Samuel, Adv Mater, 2008, 20, 3516.

[13] L. Luer, H. J. Egelhaaf, D. Oelkrug, G. Cerullo, G. Lanzani, B. H. Huisman, D. de Leeuw, Org Electron, 2004, 5, 83.

[14] O. V. Mikhnenko, H. Azimi, M. Scharber, M. Morana, P. W. M. Blom, M. A. Loi, Energ Environ Sci, 2012, 5, 6960. 
[15] H. Wang, H.-Y. Wang, B.-R. Gao, L. Wang, Z.-Y. Yang, X.-B. Du, Q.-D. Chen, J.-F. Song, H.-B. Sun, Nanoscale, 2011, 3, 2280.

[16] S. Cook, L. Y. Han, A. Furube, R. Katoh, J Phys Chem C, 2010, 114, 10962.

[17] A. J. Ward, A. Ruseckas, I. D. W. Samuel, J Phys Chem C, 2012, 116, 23931.

[18] R. J. Kline, M. D. McGehee, M. F. Toney, Nat Mater, 2006, 5, 222.

[19] D. T. Duong, M. F. Toney, A. Salleo, Phys Rev B, 2012, 86, 205205.

[20] V. Gulbinas, I. Mineviciute, D. Hertel, R. Wellander, A. Yartsev, V. Sundstrom, J Chem Phys, 2007, 127, 144907.

[21] U. Gösele, A. Seeger, Philosophical Magazine, 1976, 34, 177.

[22] A. Ruseckas, M. Theander, M. R. Andersson, M. Svensson, M. Prato, O. Inganas, V. Sundstrom, Chem Phys Lett, 2000, 322, 136.

[23] C. X. Sheng, M. Tong, S. Singh, Z. V. Vardeny, Phys Rev B, 2007, 75.

[24] J. Piris, T. E. Dykstra, A. A. Bakulin, P. H. M. van Loosdrecht, W. Knulst, M. T. Trinh, J. M. Schins, L. D. A. Siebbeles, J Phys Chem C, 2009, 113, 14500.

[25] F. Paquin, G. Latini, M. Sakowicz, P.-L. Karsenti, L. Wang, D. Beljonne, N. Stingelin, C. Silva, Phys Rev Lett, 2011, 106.

[26] F. C. Spano, J Chem Phys, 2005, 122, 234701.

[27] E. S. Manas, F. C. Spano, J Chem Phys, 1998, 109, 8087.

[28] D. Beljonne, J. Cornil, R. Silbey, P. Millie, J. L. Bredas, J Chem Phys, 2000, 112, 4749.

[29] E. V. Emelianova, S. Athanasopoulos, R. J. Silbey, D. Beljonne, Phys Rev Lett, 2010, $104,206405$.

[30] J. Gierschner, Y. S. Huang, B. Van Averbeke, J. Cornil, R. H. Friend, D. Beljonne, J Chem Phys, 2009, 130, 044105.

[31] Z. Shuai, J. L. Bredas, Phys Rev B, 2000, 62, 15452.

[32] T. S. Ahn, A. M. Muller, R. O. Al-Kaysi, F. C. Spano, J. E. Norton, D. Beljonne, J. L. Bredas, C. J. Bardeen, J Chem Phys, 2008, 128, 054505. 


\section{ADVANEED \\ ENerar \\ Submitted to MATERIALS}

[33] F. C. Spano, J. Clark, C. Silva, R. H. Friend, J Chem Phys, 2009, 130.

[34] E. Emelianova, L. J. Wang, D. Beljonne, Z. Masri, I. D. W. Samuel, in preparation.

[35] M. Surin, O. Coulembier, K. Tran, J. D. Winter, P. Leclère, P. Gerbaux, R. Lazzaroni, P. Dubois, Org Electron, 2010, 11, 767. 


\section{Submitted to MATERGY}
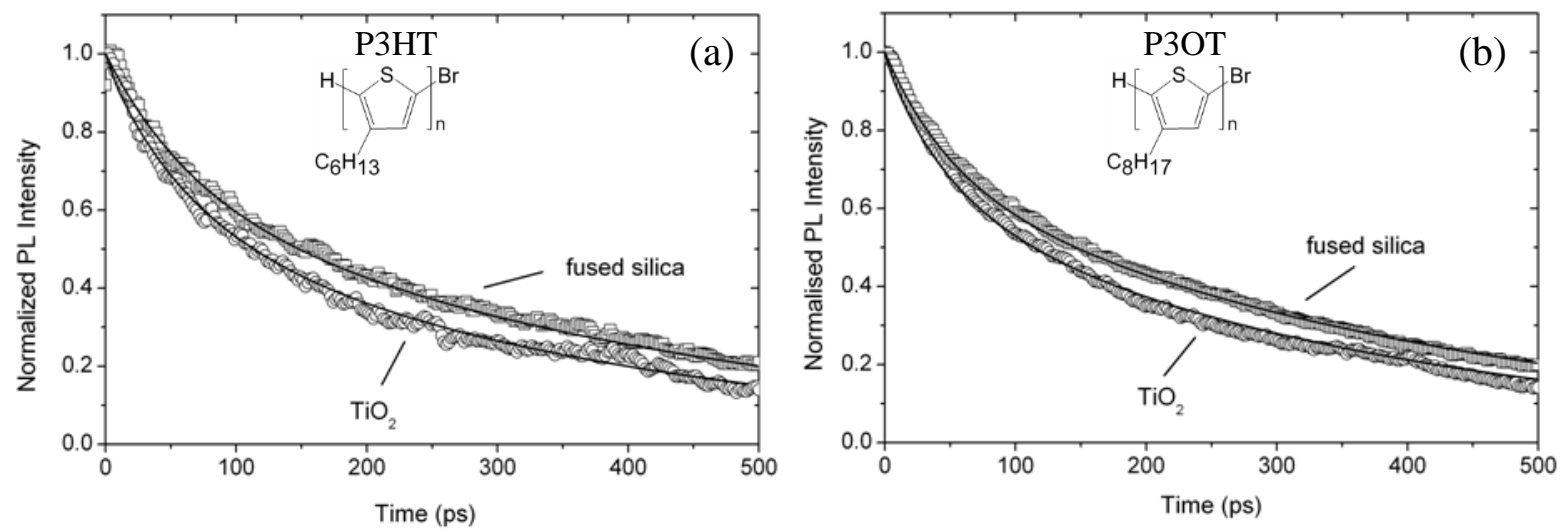

(c)

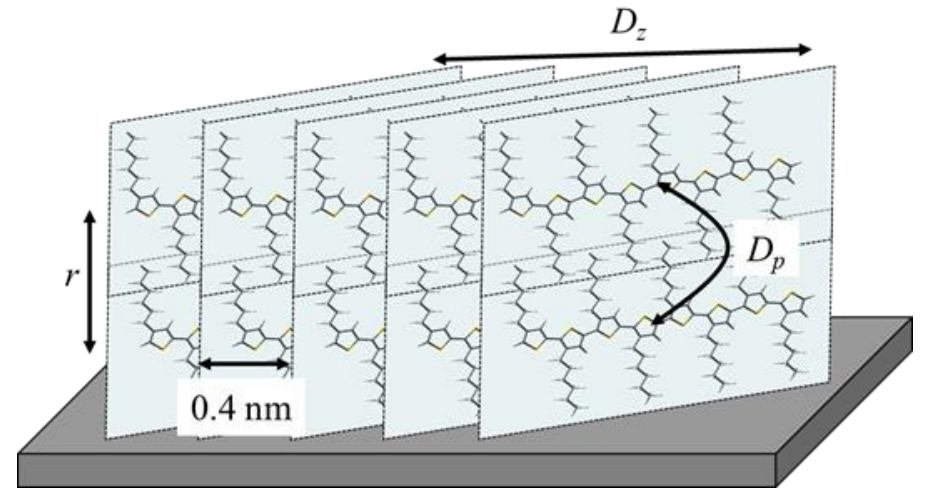

Figure 1. Fluorescence decays of (a) $\mathrm{P} 3 \mathrm{HT}$ and (b) $\mathrm{P} 3 \mathrm{OT}$ film on $\mathrm{TiO}_{2}$ and fused silica substrates. Solid lines are the fits using Equation 1 which give $D=2 \times 10^{-4} \mathrm{~cm}^{2} \mathrm{~s}^{-1}$ for P3HT and $D=1 \times 10^{-4} \mathrm{~cm}^{2} \mathrm{~s}^{-1}$ for P3OT. Insets show the chemical structures of P3HT and P3OT. Lamellae of two $\pi-\pi$ stacks of regioregular P3HT (c) with nearest neighbour cofacial separation of 0.4 $\mathrm{nm}$ and inter-stack distance $r$. Diffusion coefficient between stacks is $D_{p}$ whereas diffusion coefficient along a stack is $D_{z}$. 
Submitted to MATERIALS
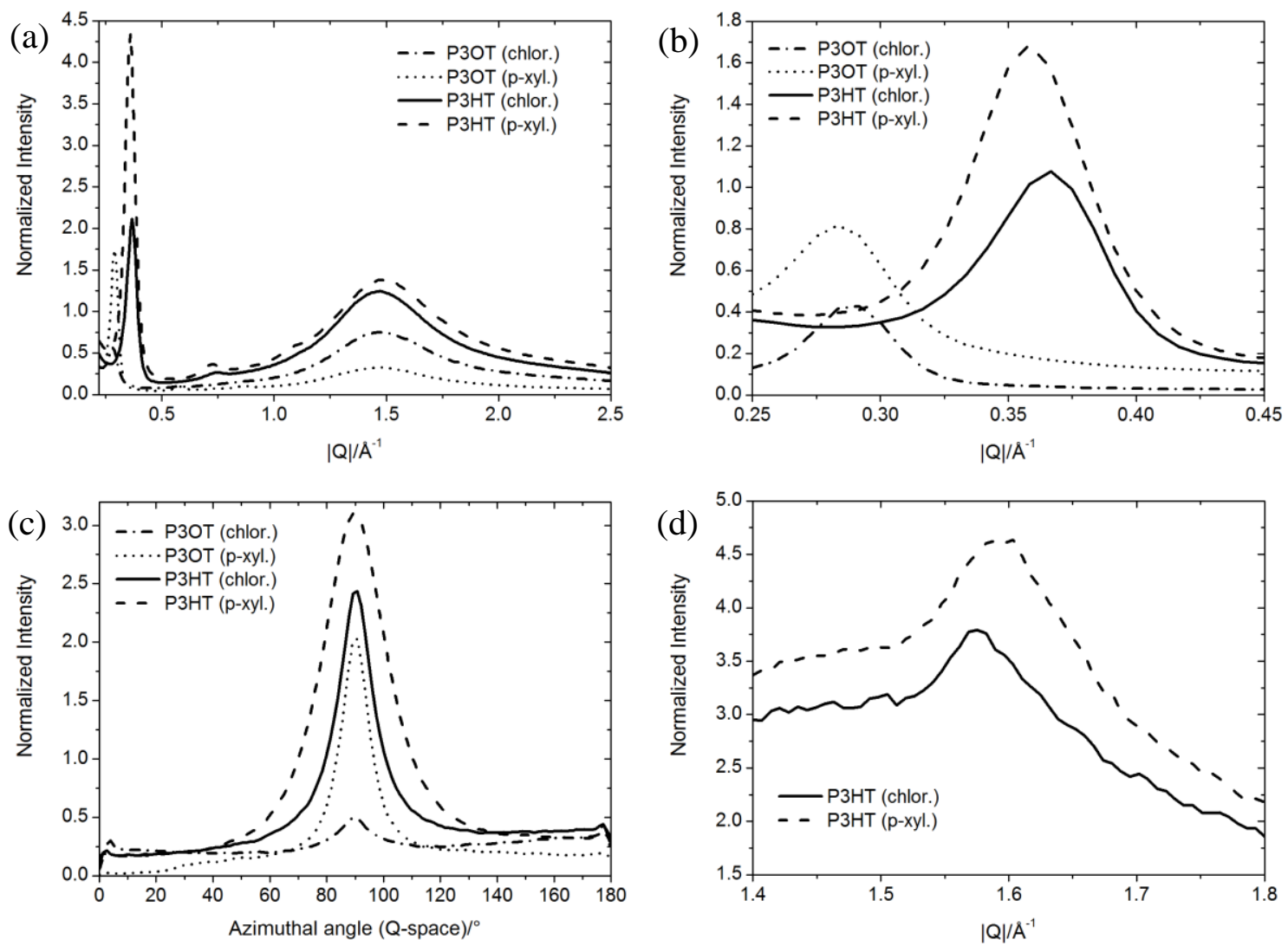

Figure 2. (a) Radial diffraction profiles in out-of-plane direction from different films of P3OT and P3HT. (b) Zoom into first order reflections. (The peaks positions as fitted by Gaussians are given in Table S1 in Supporting Information (SI)). (c) Azimuthal profiles of the first order reflections shown in (b) transformed into Q-space. Fitted values for the full width at half maximum are listed in Table S1 in SI. (d) Radial diffraction profiles in in-plane direction for P3HT films. 
(a)
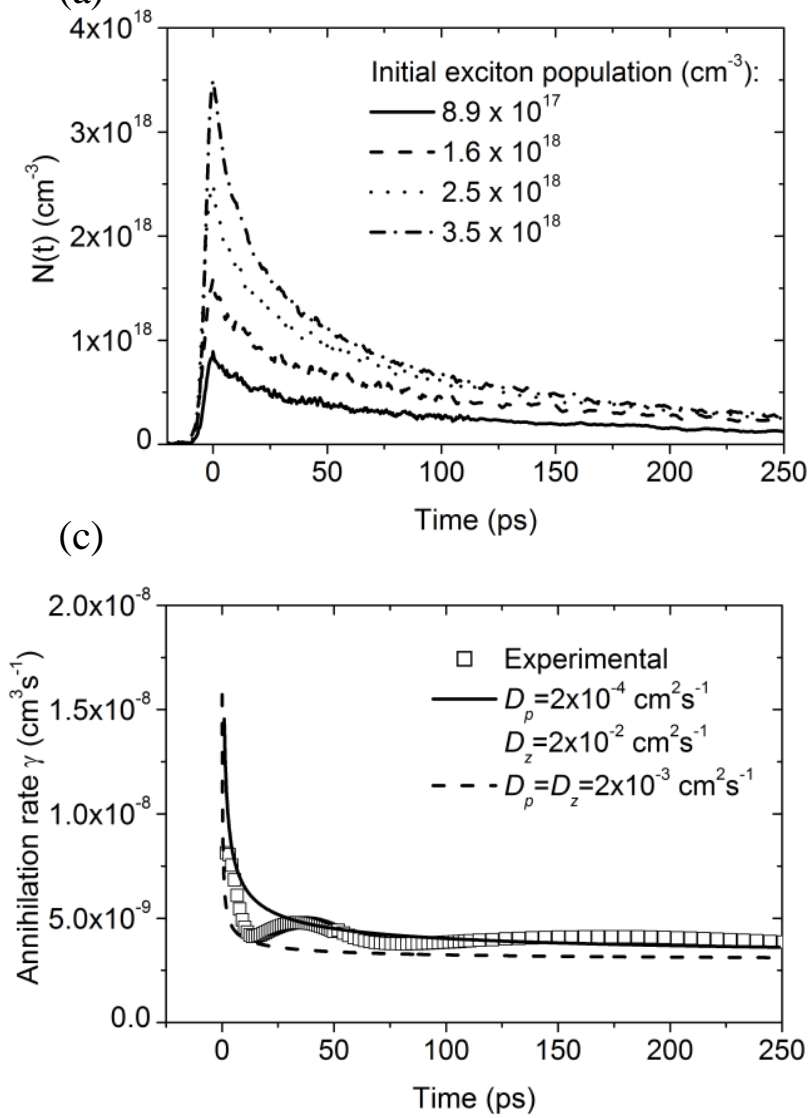

(b)

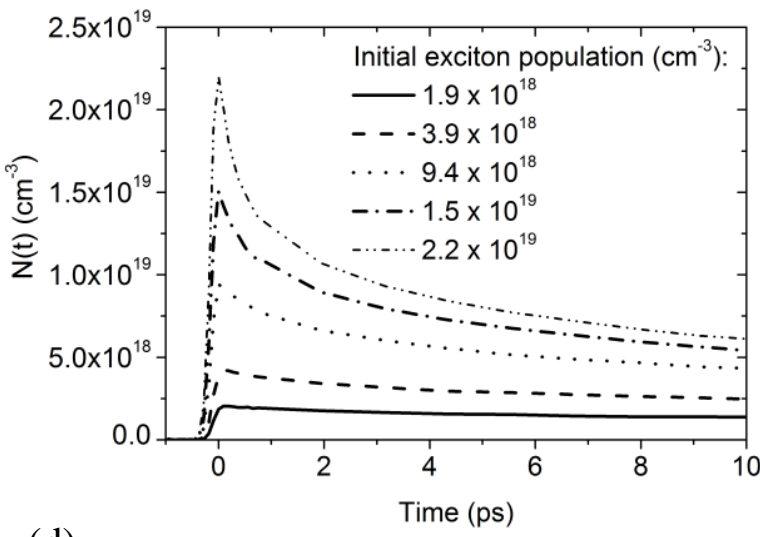

(d)

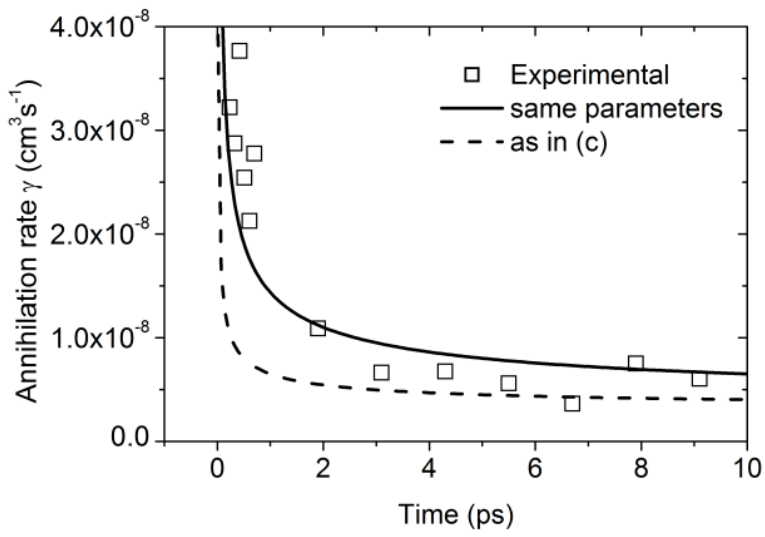

Figure 3. Time dependence of exciton population $N(t)$ in P3HT film $\left(M_{n}=76000 \mathrm{gmol}^{-1}\right)$ spincoated from chlorobenzene solution measured for different excitation densities by (a) timeresolved fluorescence after excitation at $2.2 \mathrm{eV}$ and (b) transient absorption after excitation at $2.4 \mathrm{eV}$; (c) annihilation rate obtained from time-resolved fluorescence data, (d) annihilation rate obtained from transient absorption data, solid line and dotted lines are simulated rates using Eq. 4 with $D_{p}$ and $D_{z}$ values given in the legend. 


\section{Submitted to MNERGY}

(a)

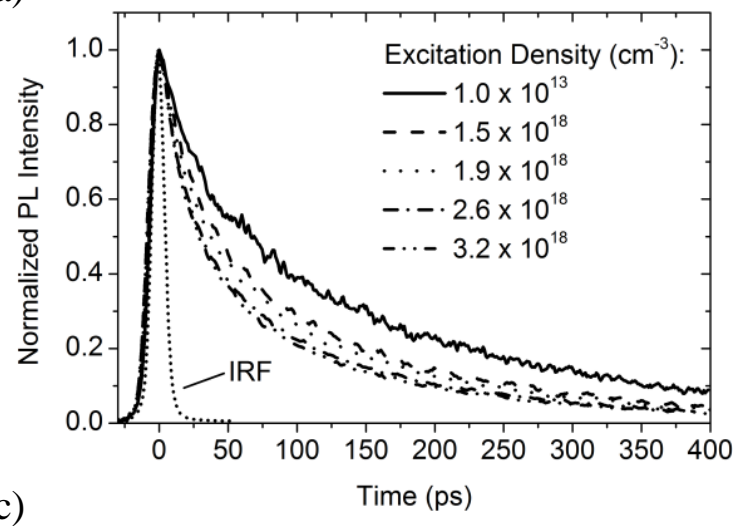

(c)

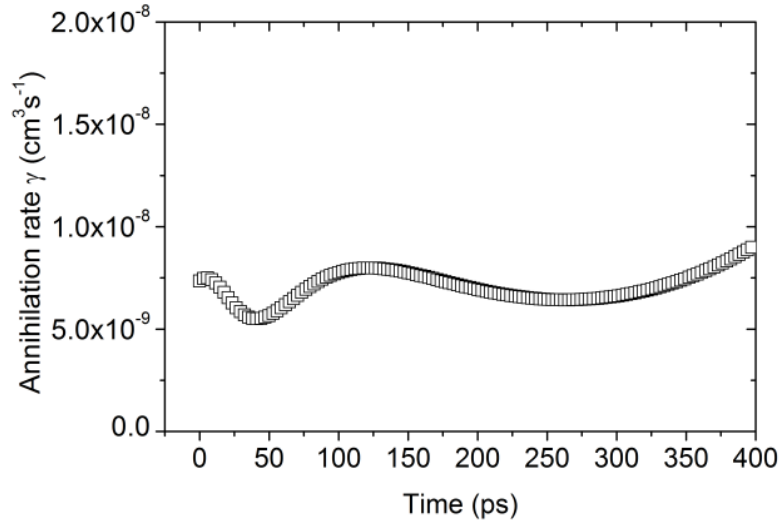

(b)

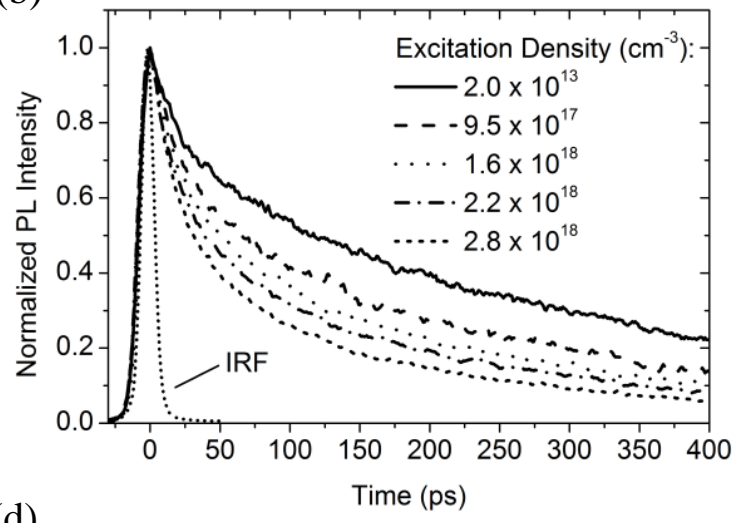

(d)

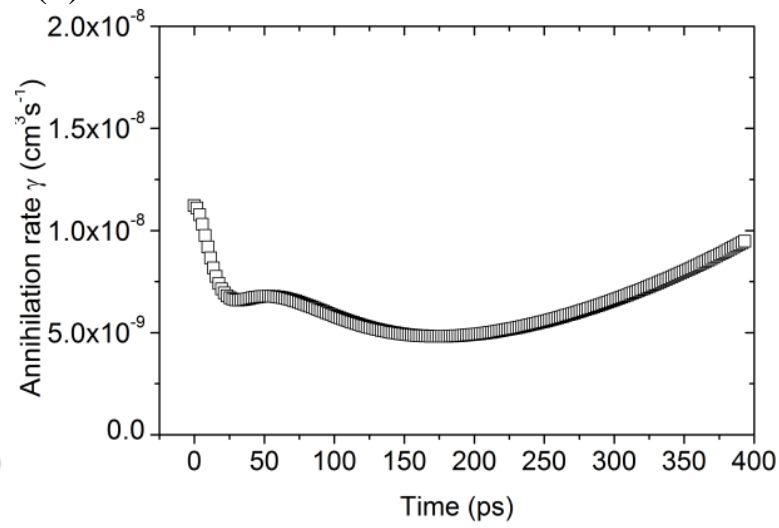

Figure 4. Fluorescence decays measured at different excitation densities for P3HT films spincoated from chlorobenzene solution after excitation at $3.1 \mathrm{eV}$ for (a) $M_{n}=13800 \mathrm{gmol}^{-1}$ and (b) $M_{n}=40000 \mathrm{gmol}^{-1}$. IRF is the instrument function. (c) and (d) show $\gamma(t)$ extracted from the PL decays shown in (a) and (b) respectively. From the $\gamma$ values between 100 ps and 300 ps we obtain $D_{\text {eff }}=4.9 \times 10^{-3} \mathrm{~cm}^{2} \mathrm{~s}^{-1}$ for $M_{n}=13800 \mathrm{gmol}^{-1}$ and $D_{\text {eff }}=3.8 \times 10^{-3} \mathrm{~cm}^{2} \mathrm{~s}^{-1}$ for $M_{n}=40000$ gmol $^{-1}$. A control experiment using $2.2 \mathrm{eV}$ and $2.45 \mathrm{eV}$ excitation gave very similar results. 


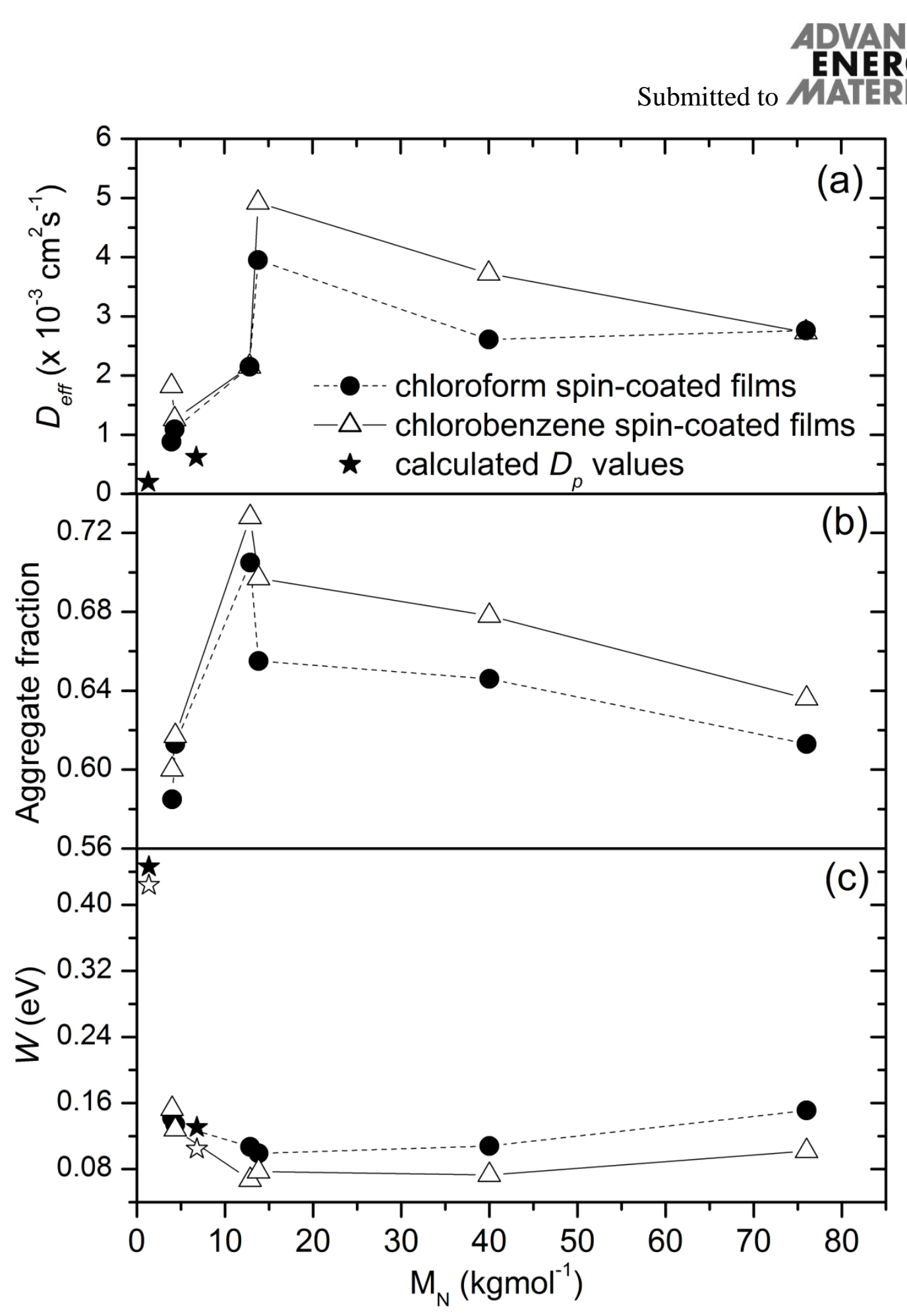

Figure 5. Variation of (a) diffusion coefficient (b) aggregate fraction and (c) exciton bandwidth as a function of $M_{n}$. Films spun from chloroform solution are represented by solid circles $(\cdots \cdots)$ whereas films spun from chlorobenzene solution are represented by open triangles $(-\triangle-)$. Calculated diffusion coefficients between stacks $D_{p}$ and exciton bandwidths for a stack size of 101 molecules are also included for two molecular weights and are denoted by solid stars $(\star)$. Exciton bandwidths for a stack size of 21 molecules have also been calculated and are represented by open stars $(\hat{\zeta})$. 


\section{Submitted to MATERIALS}
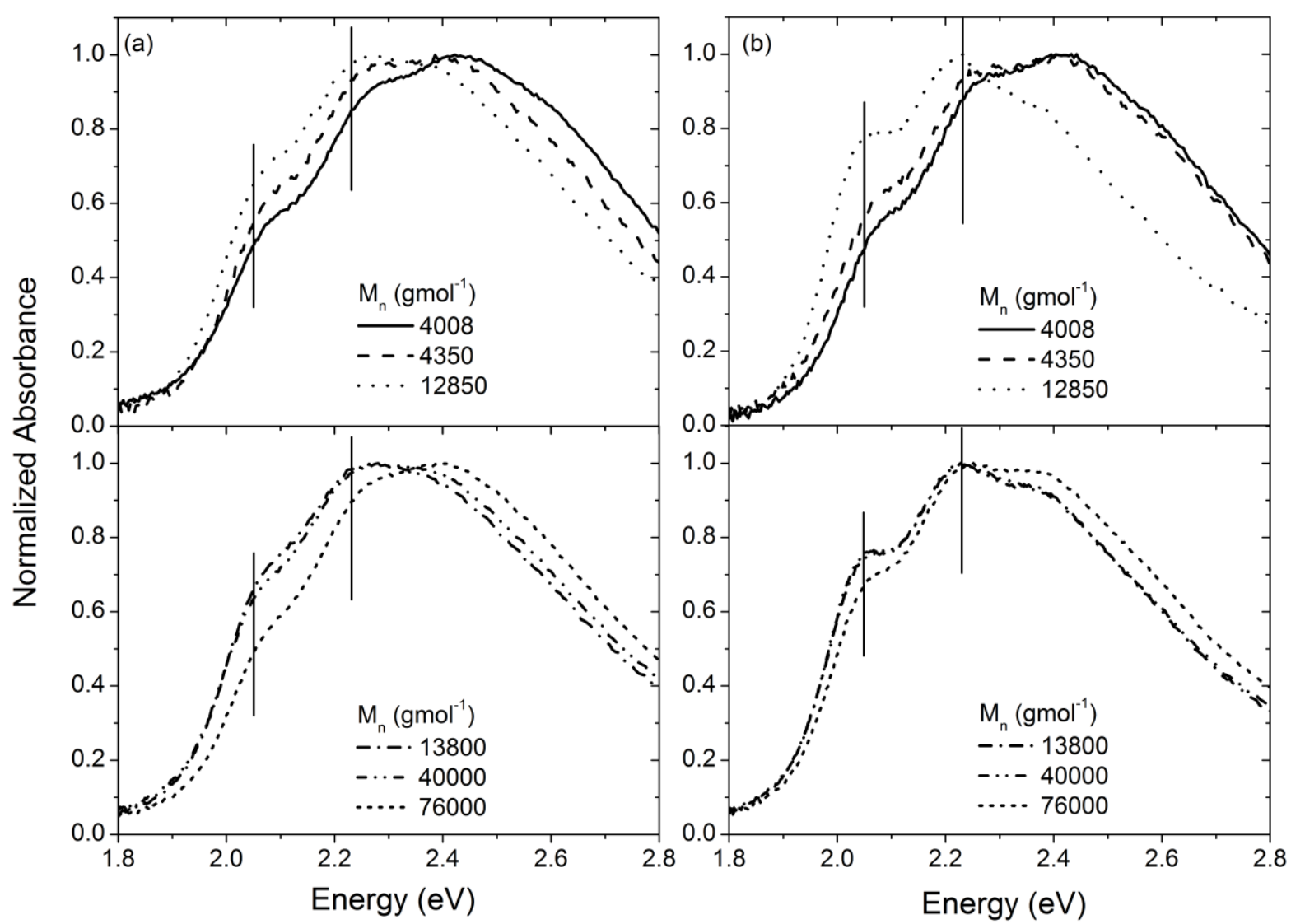

Figure 6. Normalized absorbance spectra of P3HT films spun from (a) chloroform solution and (b) chlorobenzene solution for different $M_{n}$. 

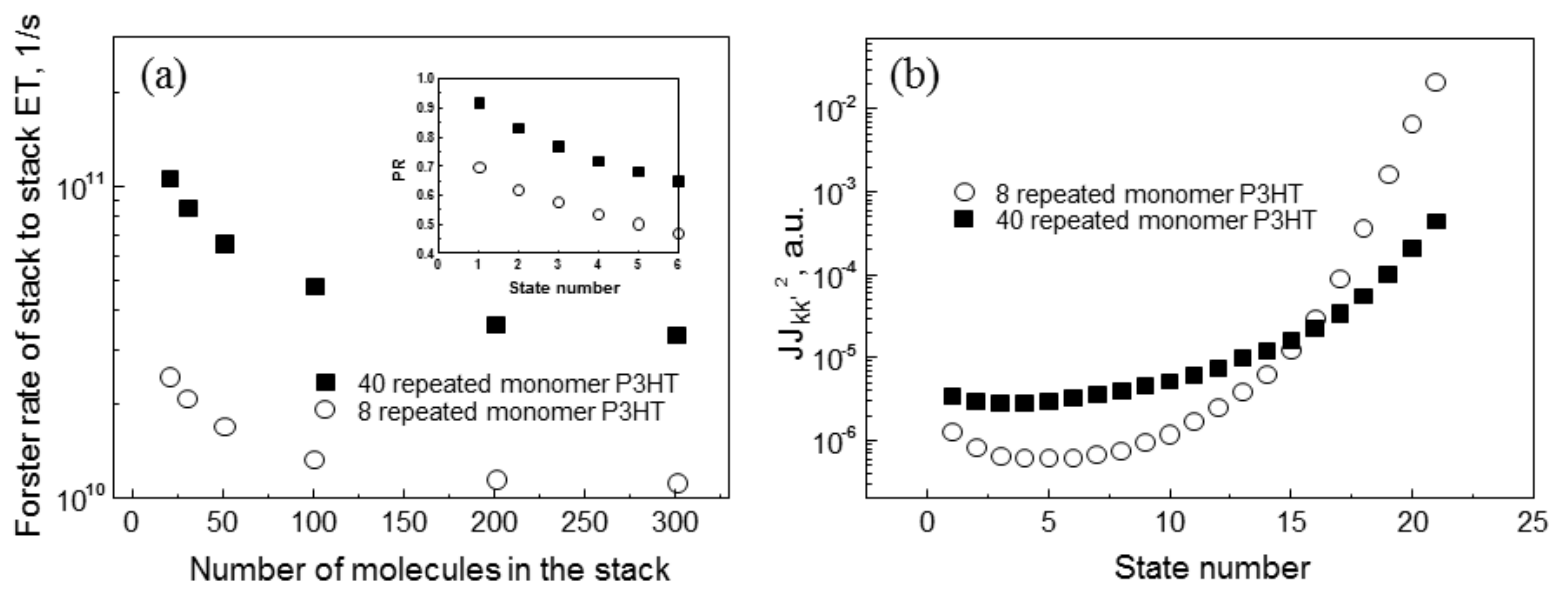

Figure 7. (a) Calculated stack-to-stack Förster energy transfer rate for the P3HT model lamellae shown for various numbers of molecules in each stack. Full squares ( $\mathbf{\square})$ correspond to P3HT molecules with 40 repeated monomers and hollow circles $(O)$ correspond to P3HT molecules with 8 repeated monomers. Insert shows the participation ratio for the 5 lowest band states for stacks of 21 molecules. (b) represents the channel input to the Förster rate from every exciton state for the corresponding model lamellae calculated for a stack size of 21 molecules. Calculations were done taking into account static Gaussian disorder with a standard deviation of $0.1 \mathrm{eV}$ and were averaged over 20000 disorder realisations. 
Table 1. Molecular weights and dispersity values $\left(\bigoplus_{M}=M_{w} / M_{n}\right)$ of all P3HT samples used along with measured results. P3HT films were spun from chloroform (1) and chlorobenzene (2) solution.

\begin{tabular}{ccccccc}
\hline Solvent & $\begin{array}{c}M_{n} \\
{\left[\mathrm{gmol}^{-1}\right]}\end{array}$ & $\bigoplus_{M}$ & $\begin{array}{c}\gamma(100 \mathrm{ps}<t<300 \mathrm{ps}) \\
{\left[\times 10^{-9} \mathrm{~cm}^{3} \mathrm{~s}^{-1}\right]}\end{array}$ & $\begin{array}{c}D_{\text {eff }} \\
{\left[\times 10^{-3} \mathrm{~cm}^{2} \mathrm{~s}^{-1}\right]}\end{array}$ & $\begin{array}{c}W \\
{[\mathrm{eV}]}\end{array}$ & $\begin{array}{c}\text { Aggregate } \\
\text { fraction }\end{array}$ \\
\hline $\mathbf{1}$ & 4008 & 1.23 & 1.3 & 0.9 & 0.142 & 0.59 \\
& 4350 & 1.17 & 1.6 & 1.1 & 0.134 & 0.61 \\
& 12850 & 1.40 & 3.1 & 2.2 & 0.107 & 0.71 \\
& 13800 & 1.20 & 5.7 & 4.0 & 0.099 & 0.66 \\
& 40000 & 1.20 & 3.8 & 2.6 & 0.108 & 0.65 \\
& 76000 & 2.10 & 4.0 & 2.8 & 0.151 & 0.61 \\
& 4008 & 1.23 & 2.6 & 1.8 & 0.153 & 0.60 \\
4350 & 1.17 & 1.8 & 1.3 & 0.128 & 0.62 \\
& 12850 & 1.40 & 3.1 & 2.2 & 0.067 & 0.73 \\
13800 & 1.20 & 7.1 & 4.9 & 0.077 & 0.70 \\
40000 & 1.20 & 5.4 & 3.8 & 0.073 & 0.68 \\
76000 & 2.10 & 4.0 & 2.8 & 0.102 & 0.64 \\
\hline
\end{tabular}


Singlet exciton diffusion in P3HT is studied by a combination of experimental and theoretical methods. We show that exciton diffusion is faster along the co-facial $\pi-\pi$ aggregates than between them. The fastest exciton diffusion is observed for intermediate molecular weight polymers and it correlates with the degree of chromophore aggregation and with exciton delocalisation along the polymer chain.

Keyword Exciton diffusion

Zarifi Masri, Arvydas Ruseckas, Evguenia V. Emelianova, Linjun Wang, Ashu K. Bansal, Andrew Matheson, Henrik T. Lemke, Martin M. Nielsen, Ha Nguyen, Olivier Coulembier, Philippe Dubois, David Beljonne, Ifor D. W. Samuel*

Molecular weight dependence of exciton diffusion in poly(3-hexylthiophene)

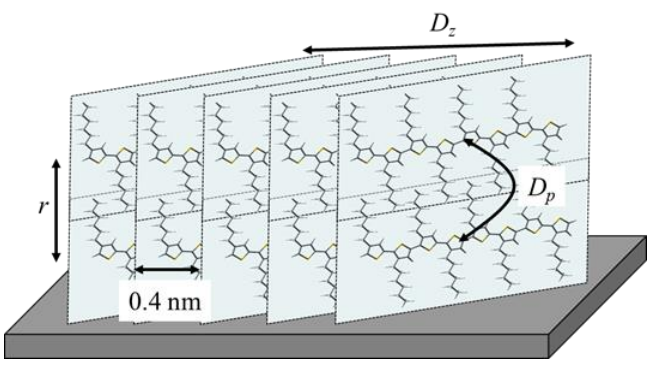


Copyright WILEY-VCH Verlag GmbH \& Co. KGaA, 69469 Weinheim, Germany, 2012.

\section{Supporting Information}

for $A d v$. Energy Mater., DOI: 10.1002/aenm.((please add manuscript number))

\section{Molecular weight dependence of exciton diffusion in poly(3-hexylthiophene)}

Zarifi Masri, Arvydas Ruseckas, Evguenia V. Emelianova, Linjun Wang, Ashu K. Bansal, Andrew Matheson, Henrik T. Lemke, Martin M. Nielsen, Ha Nguyen, Olivier Coulembier, Philippe Dubois, David Beljonne, Ifor D. W. Samuel*

\section{Surface quenching measurements on tethered $\mathbf{C}_{60}$}

Time-resolved fluorescence measurements were performed using the time-correlated single photon counting (TCSPC) technique. An excitation of $393 \mathrm{~nm}$ with a pulse width at fwhm of about 200 ps from a pulsed Picoquant GaN was used. Measurements were performed under a nitrogen atmosphere.
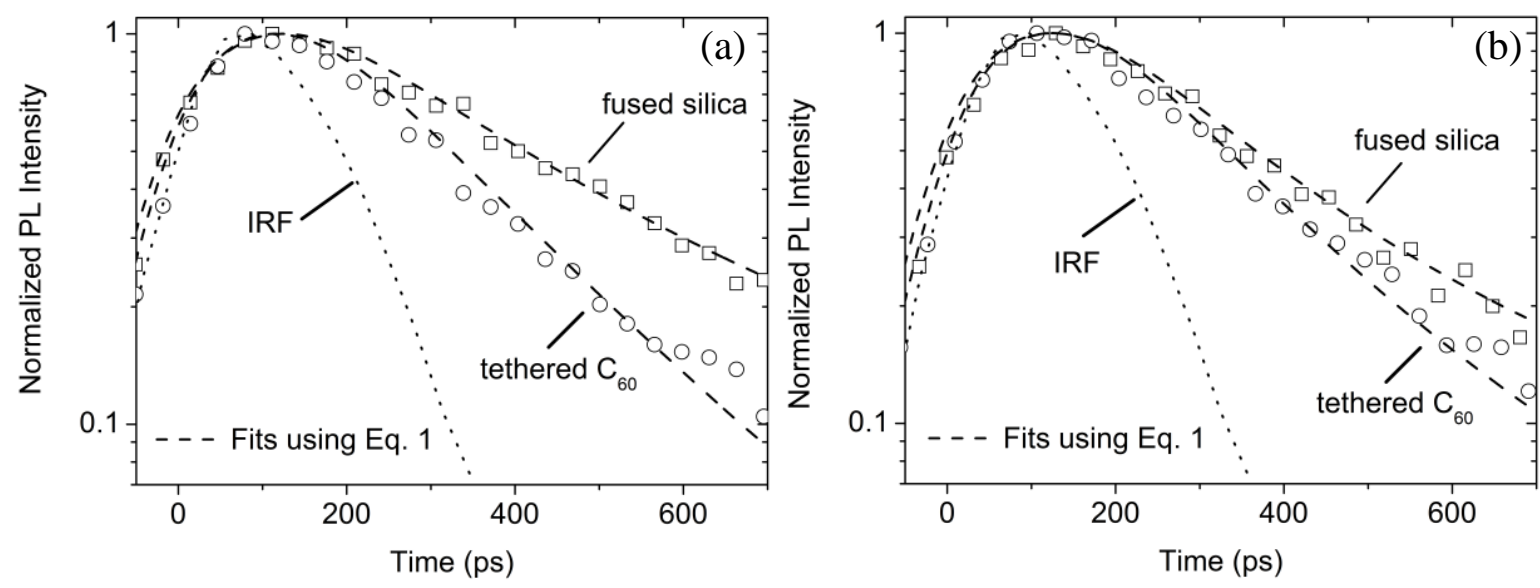

Figure S1: Fluorescence decays of (a) P3HT and (b) P3OT film on tethered $\mathrm{C}_{60}$ and fused silica substrates with fits obtained using Equation 1 which give $D=4 \times 10^{-4}$ for P3HT and $D=2 \times 10^{-4} \mathrm{~cm}^{2} \mathrm{~s}^{-1}$ for P3OT. IRF is the instrument response function. 


\section{X-ray diffraction (XRD) experiments}

X-ray diffraction experiments were performed at the X-ray centre at Risø DTU on $80 \mathrm{~nm}$ thick films of P3HT $M_{n}=13800 \mathrm{gmol}^{-1}$ and P3OT $M_{n}=13500 \mathrm{gmol}^{-1}$. The out of plane stacking distances as determined from the first order reflection are $20.3 \AA$, $19.6 \AA, 16.5 \AA, 16.1 \AA$ for P3OT (chloroform), P3OT (p-xylene), P3HT (chloroform) and P3HT (p-xylene), respectively. The grazing incidence geometry was employed to suppress background scattering from the substrate by selecting an incidence angle below the angle of total external reflection from the substrate but above the angle of total external reflection from the film, thus maximizing the signal from the film. By nature of the measurements, the out of plane direction is measured with a slight offset. This is also seen as the lack of data points around the $90^{\circ}$ azimuth angle in Figure 2 (c).

The peak radial positions as fitted by Gaussians are given in Table S1. The fitted values for the full width at half maximum for the first order reflections (azimuthal) are also listed in the table. Using fitted values eliminates possible offsets introduced by measuring only part of the out of plane peak profiles, as discussed above.

Table S1: Radial positions and azimuthal widths of the first order out-of-plane reflections

\begin{tabular}{|lcc|}
\hline Sample & Radial peak position $/ \AA^{\mathbf{1}}$ & Azimuthal peak width ' $^{\circ}$ \\
\hline P3OT (chlor.) & 0.31 & 13.7 \\
P3OT (p-xyl.) & 0.32 & 9.7 \\
P3HT (chlor.) & 0.38 & 12.5 \\
P3HT (p-xyl.) & 0.39 & 25.9 \\
\hline
\end{tabular}




\section{Calculation of aggregate fraction}

The aggregate fraction or fraction of film made of planarized and $\pi$-stacked polymer chains is estimated by subtracting the dilute solution absorption spectra from the film absorption spectra. As the amorphous phase is made of disordered polymer chains, the absorbance due to these unaggregated polymers can simply be approximated by the absorption spectra from the dilute solution.

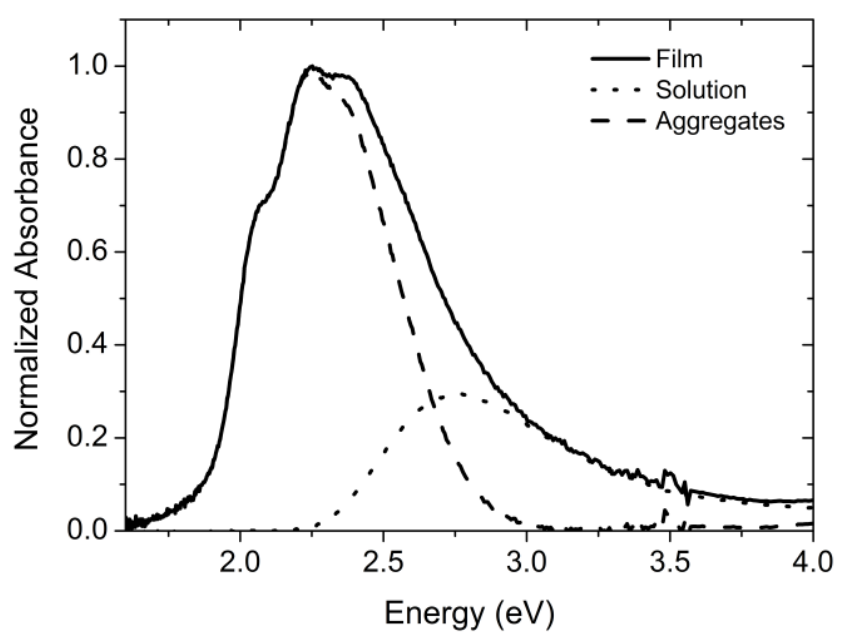

Figure S2: Representative absorbance spectra showing calculation of aggregate fraction.

The aggregate fraction is thus calculated by taking the ratio of the area under the aggregate and film absorbance curves (Figure S2):

$$
\text { Fraction of aggregates }=\frac{\text { Area of } A b s_{\text {Crys }}}{\text { Area of } A b s_{\text {Film }}}
$$

To account for the increase in oscillator strength going from a non-planar chain in the amorphous phase to a planarized chain in the crystalline aggregates, a relative oscillator strength of $39 \%$ obtained by Clark et al. ${ }^{[11]}$ was used. 